\title{
Riboswitch-based sensor in low optical background
}

\author{
Svetlana V. Harbaugh, Molly E. Davidson, Yaroslav G. Chushak*, Nancy Kelley-Loughnane, and \\ Morley O. Stone \\ Air Force Research Laboratory, Human Effectiveness Directorate, Applied Biotechnology Branch, \\ Wright-Patterson Air Force Base, Dayton, OH 45433-5707 \\ *Biotechnology HPC Software Applications Institute, Telemedicine and Advanced Technology \\ Research Center, U.S. Army Medical Research and Material Command, \\ Fort Detrick, MD 21702, USA
}

\begin{abstract}
Riboswitches are a type of natural genetic control element that use untranslated sequence in the RNA to recognize and bind to small molecules that regulate expression of that gene. Creation of synthetic riboswitches to novel ligands depends on the ability to screen for analyte binding sensitivity and specificity. In our work, we have coupled a synthetic riboswitch to an optical reporter assay based on fluorescence resonance energy transfer (FRET) between two geneticallycoded fluorescent proteins. Specifically, a theophylline-sensitive riboswitch was placed upstream of the Tobacco Etch Virus (TEV) protease coding sequence, and a FRET-based construct, BFP-eGFP or eGFP-REACh, was linked by a peptide encoding the recognition sequence for TEV protease. Cells expressing the riboswitch showed a marked optical difference in fluorescence emission in the presence of theophylline. However, the BFP-eGFP FRET pair posses significant optical background that reduces the sensitivity of a FRET-based assay. To improve the optical assay, we designed a nonfluorescent yellow fluorescent protein (YFP) mutant called REACh (for Resonance Energy-Accepting Chromoprotein) as the FRET acceptor for eGFP. The advantage of using an eGFP-REACh pair is the elimination of acceptor fluorescence which leads to an improved detection of FRET via better signal-to-noise ratio. The EGFP-REACh fusion protein was constructed with the TEV protease cleavage site; thus upon TEV translation, cleavage occurs diminishing REACh quenching and increasing eGFP emission resulting in a 4.5-fold improvement in assay sensitivity.
\end{abstract}

Keywords: Riboswitch, theophylline, TEV protease, FRET, REACh

\section{INTRODUCTION}

Riboswitches are regulatory RNAs that are located at the 5'-untranslated regions (UTRs) of certain mRNA. ${ }^{1-4}$ These RNA-based sensors bind to a ligand and alter the gene expression of downstream genes. Riboswitches are conceptually divided into two parts: an aptamer and an expression platform. The aptamer directly binds the small molecule, and the expression platform undergoes structural changes in response to the changes in the aptamer and regulates gene expression. Riboswitches have been studied for their ability to sense both natural and non-natural ligands and to display these changes via their gene expression platform. Recently, Gallivan and coworkers have developed a synthetic theophylline riboswitch that shows a 36-fold increase in protein expression in the presence of ligand and a very low level of expression in its absence. ${ }^{5}$ Their expression platform included the reporter gene encoding $\beta$-galactosidase. In order to develop an optical screening assay for synthetic riboswitches, we have coupled the theophylline-sensitive riboswitch to a reporter assay based on fluorescence resonance energy transfer (FRET) between two genetically-coded fluorescent proteins. The first protein in the fluorescent protein pair acts as the electron donor and the second represents the electron acceptor. FRET involves the distance-dependent energy transfer from an excited donor molecule to a lower energy acceptor and is measured in terms of spectral changes in the emission signal. Due to the attractive attributes the application of FRET to GFP and GFP variants has become a powerful tool to detect a broad range of molecular events including enzymatic turnover of substrates and binding interactions. ${ }^{6-9}$ We have constructed two recombinant intramolecular FRET-based protein pairs. The first construct is based on the widely used BFP-eGFP pair, ${ }^{7,} 10-12$ and the second is composed of eGFP and a nonfluorescent yellow fluorescent protein (YFP) mutant called REACh (for Resonance Energy-Accepting Chromoprotein). ${ }^{9}$ Two fluorescent proteins have been linked with a 17 amino acid peptide sequence that includes the recognition site of Tobacco Etch Virus (TEV) protease which cleaves with a high specificity ${ }^{13}$

Nanobiosystems: Processing, Characterization, and Applications, edited by Emily M. Heckman, Thokchom Birendra Singh, Junichi Yoshida, Proc. of SPIE Vol. 7040, 70400C, (2008) · 0277-786X/08/\$18 · doi: 10.1117/12.796122 
and has been a well characterized mutant for expression in E. coli. ${ }^{14}$ To build a riboswitch-based sensor, we have placed a synthetic theophylline riboswitch upstream of the TEV protease encoding sequence. Thus, in the presence of theophylline the riboswitch is activated (5' UTR RNA conformational rearrangement) leading to translation of the TEV transcript, producing TEV protease which can cleave the FRET pair resulting in the spectral changes of the emission signal. We have tested the riboswitch construct in E. coli cells. In the presence of theophylline, cells expressing the riboswitch and BFP-eGFP protein pair showed a marked decrease of the acceptor fluorescence at $510 \mathrm{~nm}$ and increase of donor fluorescence at $445 \mathrm{~nm}$, as shown schematically in Figure 1a. In this case, the ratio of emission at 510/445 nm was used to quantify the performance of the riboswitch. E. coli cells expressing riboswitch and eGFP-REACh protein pair showed significant increase of eGFP emission in the presence of theophylline (Figure 1b). Since the fluorescence of REACh is eliminated this "dark" protein does not show any emission characteristics (before or after FRET cleavage). In this case, the eGFP emission at $510 \mathrm{~nm}$ was used to quantify the activation of riboswitch.

In this study we have compared two FRET-based reporting systems for detection of theophylline sensitive riboswitch activation. We have shown the advantage of using an eGFP-REACh pair as a reporter in optical screening assay for synthetic riboswitches.

a.

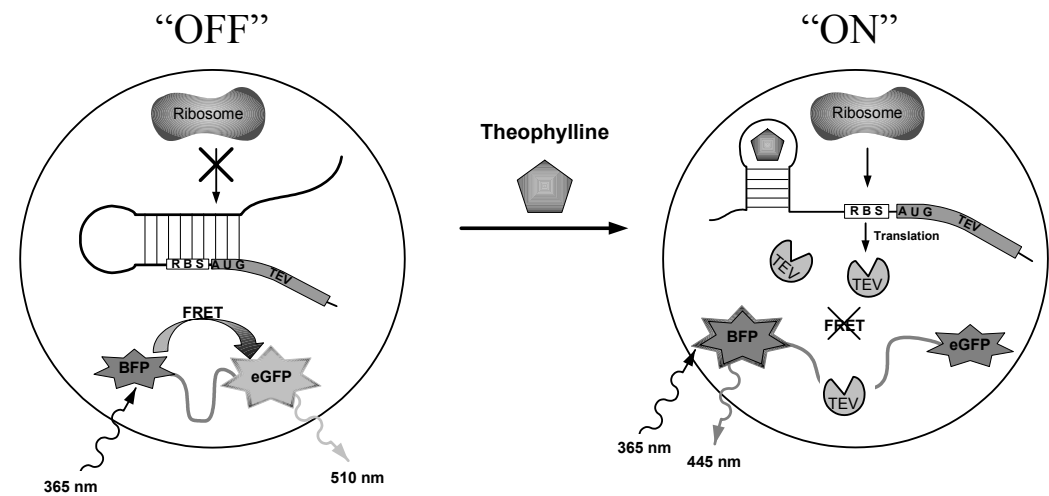

b.
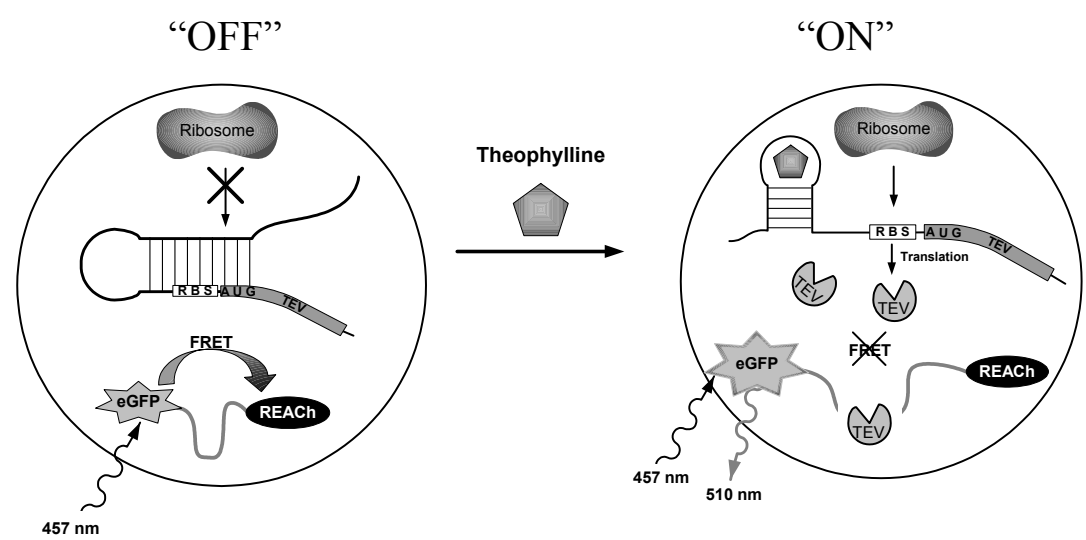

Fig. 1. FRET-based reporter assay for detection of theophylline-responsive riboswitch activation in vivo. In the absence of theophylline, extensive pairing of riboswitch with ribosome binding site (RBS) prevents translation of TEV protease, and riboswitch is in "OFF" state. Addition of theophylline changes the conformation of the riboswitch unpairing the RBS, initiating translation, i.e., riboswitch is in "ON" state. Synthesized TEV protease cleaves the linker in the FRET-based fusion protein causing a change in the fluorescence signal. (a) In the absence of theophylline cells expressing riboswitch-TEV protease construct and BFP-(tev linker)-eGFP show emission at $510 \mathrm{~nm}$ when excited at $365 \mathrm{~nm}$. In the presence of theophylline, the emission at $510 \mathrm{~nm}$ is decreased with concurrent increase in emission at $445 \mathrm{~nm}$ as a result of FRET-protein cleavage. (b) Cells expressing riboswitch-TEV protease construct and eGFP-(tev linker)-REACh do not show significant emission in the absence of theophylline upon excitation at $457 \mathrm{~nm}$. In the presence of theophylline the emission at $510 \mathrm{~nm}$ is increased as a result of fusion protein cleavage and loss of FRET-based quenching. 


\section{EXPERIMENTAL}

\subsection{Materials}

Isopropyl- $\beta$-D-thiogalactopyranoside (IPTG), kanamycin, chloramphenicol, ampicillin, and theophylline were obtained from Sigma Chemical Company (St. Louis, MO). L-(+) rhamnose monohydrate and dimethylsulfoxide (DMSO) were purchase from Fluka (Switzerland). Plasmid Miniprep, MinElute PCR purification kit, and QIAEX II Gel Extraction Kit were purchased from Qiagen (Valencia, CA). DifcoTM Luria-Bertani (LB) was purchased from Becton Dikinson and Company (Sparks, MD). Synthetic oligonucleotodes were supplied by Integrated DNA Technologies (Coralville, IA), and the construct of the synthetic theophylline riboswitch upstream of the TEV protease gene was made by GeneScript (CA). All restriction enzymes and T4 DNA-ligase were obtained from Invitrogen. Pfu Ultra DNA-polymerase was purchased from Stratagene (La Jolla, CA). MAX Efficiency ${ }^{\circledR}$ DH5 $\alpha^{\mathrm{TM}}$, BL21 (DE3), and One Shot ${ }^{\circledR}$ TOP10 competent E. coli cells were acquired from Invitrogen. The tobacco etch virus protease gene construct and plasmid pHWG640 were the generous gifts from Dr. Helena Berglund from Karolinska Institute in Stockholm, Sweden and Dr. Josef Altenbuchner from the Institute of Industrial Genetics at the University of Stuttgart, Germany, respectively. The plasmid vector pSAL8.1 was kindly provided by Dr. Justin Gallivan from Emory University, Atlanta, GA.

\subsection{Plasmid Construction and Recombinant Protein Purification}

The experiments involving examination of riboswitch activity via TEV protease cleavage of BFP-(tev linker)-eGFP or eGFP-(tev linker)-REACh proteins in E. coli required the construction of several expression vectors. The TEV linker is a 17 amino acid sequence (SLYKKAGSENLYFQSGT) containing the TEV protease cleavage sequence (underlined). The TEV protease gene was cloned downstream of the synthetic riboswitch sequence based on published work by Lynch et al. ${ }^{5}$ The theophylline riboswitch-TEV protease construct with restriction sites Xba I and Hind III was cloned into the pET28a plasmid, and the theophylline riboswitch-TEV construct with restriction sites Kpn I and Hind III was cloned into the pSAL plasmid with the corresponding sites — the resulting vectors was designated as pET28a:rsTEV and pSAL:rsTEV, respectively. The TEV protease gene without the upstream riboswitch sequence was also cloned into the pET28a (i.e., pET28a:TEV) or into pSAL (i.e., pSAL:TEV) vector. The mutation C151A that yields to inactive TEV protease was prepared using the QuikChange ${ }^{\mathbb{B}}$ II Site-Directed Mutagenesis kit (Stratagene) using pSAL:rsTEV as a template. Lac-promoter directed expression of the TEV protease was performed using pET28a:rsTEV and pET28a:TEV. The tac-promoter directed expression of the TEV protease was performed using pSAL:rsTEV, pSAL:TEV, and pSAL:TEVC151A. The Y145W mutation that yields REACh construct was introduced into the YFP sequence via sitedirected mutagenesis kit. The BFP-(tev linker)-eGFP or eGFP-(tev linker)-REACh fusion protein was cloned via the restriction sites Nde I and Hind III into the pHWG640 vector which utilizes a rhamnose promoter for high protein expression. pET28a:rsTEV and pHWG640:BFP-(tev linker)-eGFP or pET28a:TEV and pHWG640:BFP-(tev linker)eGFP were co-transformed into E. coli BL21(DE3) cells. pSAL:rsTEV and pHWG640:eGFP-(tev linker)-REACh or pSAL:TEV and pHWG640:eGFP-(tev linker)-REACh were co-transformed into E. coli TOP10 cells.

All plasmid manipulations were performed using the standard cloning techniques, ${ }^{15}$ and the sequences of all constructs have been verified by DNA sequencing at the Plant-Microbe Genomics Facility of the Ohio State University.

To generate purified recombinant BFP-(tev linker)-eGFP, eGFP-(tev linker)-REACh, and TEV protease, these proteins were cloned with $6 x$ His tag and expressed in E. coli BL21(DE3) cells. His-tagged fusion proteins were purified according to standard technique. ${ }^{16} \mathrm{TEV}$ protease was purified as described. ${ }^{14}$

\subsection{Proteolytic Assay in Bacteria}

Cultures of BL21 cells with plasmids pHWG640:BFP-(tev linker)-eGFP and a TEV protease expressing plasmid, either pET28a:TEV or pET28a:rsTEV, in $150 \mathrm{ml}$ of LB supplemented with kanamycin $(25 \mu \mathrm{g} / \mathrm{ml})$ and chloramphenicol $(25$ $\mu \mathrm{g} / \mathrm{ml}$ ) were grown for 6 hours after a $1 \%$ inoculation from an overnight culture. The cultures were supplemented with two inducers. First $0.4 \%$ rhamnose was added at $\mathrm{OD}_{600}$ of $0.7-0.8$ and $30 \mathrm{~min}$ after the cells were induced with $0.5 \mathrm{mM}$ IPTG. After 30 min of induction with IPTG, several cultures harboring pHWG640:BFP-(tev linker)-eGFP and pET28a:TEV (+/- riboswitch) were treated with either $2.5 \mathrm{mM}$ Theophylline in DMSO, or an equivalent volume of DMSO for controls with no analyte. The cultures were incubated at $37^{\circ} \mathrm{C}$ and shaken at $200 \mathrm{rpm}$. Aliquots of $10 \mathrm{ml}$ 
culture were collected in $15 \mathrm{ml}$ Falcon tubes at $0,60,120,180,240,300$, and $360 \mathrm{~min}$. Cells were harvested by centrifugation at $4,000 \mathrm{x} \mathrm{g}$ in Thermo Electron CL3R centrifuge for 15 min at $4{ }^{\circ} \mathrm{C}$ and cell pellets stored at $-80^{\circ} \mathrm{C}$ for future processing.

Cultures of TOP10 cells with plasmids pHWG640:eGFP-(tev linker)-REACh and a TEV protease expressing plasmid, either pSAL:rsTEV or pSAL:TEV, in $150 \mathrm{ml}$ of LB supplemented with ampicillin (100 $\mu \mathrm{g} / \mathrm{ml})$ and chloramphenicol $(25$ $\mu \mathrm{g} / \mathrm{ml}$ ) were grown at $37{ }^{\circ} \mathrm{C}$ to an $\mathrm{OD}_{600}$ of $0.4-0.5$ and induced with $0.4 \%$ rhamnose for pHWG640:eGFP-(tev linker)REACh expression. After 30 min of induction, several cultures harboring controls or riboswitch were treated with either $2.5 \mathrm{mM}$ Theophylline in DMSO, or an equivalent volume of DMSO. Cells were grown at $37{ }^{\circ} \mathrm{C}$ with shaking at $225 \mathrm{rpm}$ for 6 hours. Aliquots of $10 \mathrm{ml}$ culture were collected in $15 \mathrm{ml}$ Falcon tubes at 0,60, 120, 180, 240, 300, and $360 \mathrm{~min}$. Cells were harvested by centrifugation at 4,000 $\mathrm{x} g$ in Thermo Electron CL3R centrifuge for 10 min at $4{ }^{\circ} \mathrm{C}$.

The cell pellets were resuspended in $0.4 \mathrm{ml}$ of the buffer (100 mM Tris- $\mathrm{HCl}, \mathrm{pH} 8.0 / 150 \mathrm{mM} \mathrm{NaCl} / 1 \mathrm{mM} \mathrm{MgCl} 2)$ and incubated on ice for 30 minutes. The buffer was supplemented with Lyzosome $(0.1 \mathrm{mg} / \mathrm{mL})$, Benzonase ${ }^{\circledR}$ Nuclease $(10$ $\mu \mathrm{L} / 10 \mathrm{~mL}$ ), and the protease inhibitor iodoacetamide $(8 \mathrm{mM})$. The samples were clarified by centrifugation at $14000 \mathrm{xg}$ for 30 minutes at $4^{\circ} \mathrm{C}$ in an Eppendorf 5417R centrifuge (Fisher Scientific, Fairlawn, NJ, USA). The clarified cellular extracts were examined using spectrofluorimetric analyses.

\subsection{Spectral Analysis}

Emission spectra of recombinant BFP-(tev linker)-eGFP and eGFP-(tev linker)-REACh were acquired by utilizing Molecular Devices Spectra Max M5 Spectrophotometer. Upon excitation at $365 \mathrm{~nm}$ the constructed BFP-(tev linker)eGFP FRET pair shows emission peak at $450 \mathrm{~nm}$ for the donor BFP and emission peak at $510 \mathrm{~nm}$ for the acceptor eGFP. Proteolyc cleavage of FRET protein by TEV protease resulted in a shift of relative intensity of the emission peaks with the loss of emission intensity at $510 \mathrm{~nm}$ and increase of intensity at $450 \mathrm{~nm}$ (Figure 2a). The emission ratio of $510 \mathrm{~nm}$ $1450 \mathrm{~nm}$ was used to quantify the activity of constructed riboswitch.

Samples containing recombinant eGFP-(tev linker)-REACh FRET-based protein were excited at $457 \mathrm{~nm}$ and showed only insignificant emission peak at $510 \mathrm{~nm}$ for the donor eGFP. Proteolytic cleavage of FRET protein by TEV protease resulted in increase of eGFP emission at $510 \mathrm{~nm}$ (Figure 2b). The emission at $510 \mathrm{~nm}$ was used to quantify the activity of constructed riboswitch.

\section{RESULTS AND DISCUSSION}

The earliest use of GFP and GFP variants as FRET pairs was in the genetic construction of FRET-based protease substrates ${ }^{17}, 18$. Linking of two genetically encoded fluorescent proteins by a peptide containing the protease cleavage sequence allows detection of protease activity in living cells. In our work, we designed two FRET-based substrates for detection of TEV protease activity in cells under control of a theophylline-responsive riboswitch. The FRET pairs, BFP and eGFP or eGFP and REACh, was fused by a 17 amino acids spacer peptide. The linker contains a 7 amino acids TEV protease recognition peptide and a flanking spacer arm to increase its accessibility to the TEV protease. The first FRET pair tested was BFP-(tev linker)-eGFP protein. When recombinant BFP-(tev linker)-eGFP protein was selectively excited at $365 \mathrm{~nm}$, which is optimal for BFP donor, the eGFP acceptor showed emission at $510 \mathrm{~nm}$ as a result of FRET. Incubation of BFP-(tev linker)-eGFP with TEV protease resulted in a decrease of the eGFP emission and a slight increase in BFP emission at $445 \mathrm{~nm}$ (Figure 2a). To quantify the FRET efficiency of uncleaved versus cleaved substrate, we used the ratio of an acceptor ( $510 \mathrm{~nm}$ for eGFP) emission to donor fluorescence ( $445 \mathrm{~nm}$ for BFP). It was determined that complete cleavage of the BFP-(tev linker)-eGFP substrate destroyed FRET, leading to a 5.2-fold change in the 510 $\mathrm{nm}$ : $445 \mathrm{~nm}$ emission ratio. Thus, when using the BFP-(tev linker)-eGFP FRET pair, one must calculate the $510 \mathrm{~nm}$ : $445 \mathrm{~nm}$ emission ratio to determine activity.

The second FRET pair composed of an eGFP donor and REACh (for Resonance Energy-Accepting Chromoprotein) acceptor showed a small emission signal at $510 \mathrm{~nm}$ when excited at $457 \mathrm{~nm}$ (which is optimal for eGFP). Incubation 
with TEV protease resulted in a 3.4-fold increase in eGFP emission (Figure 2b). By eliminating REACh acceptor fluorescence, the detecting of TEV protease activity, and thus assay sensitivity, increased dramatically. Using an eGFP(tev linker)-REACh substrate, we were able to determine TEV cleavage activity by measuring emission at $510 \mathrm{~nm}$ without any additional spectroscopic manipulation.
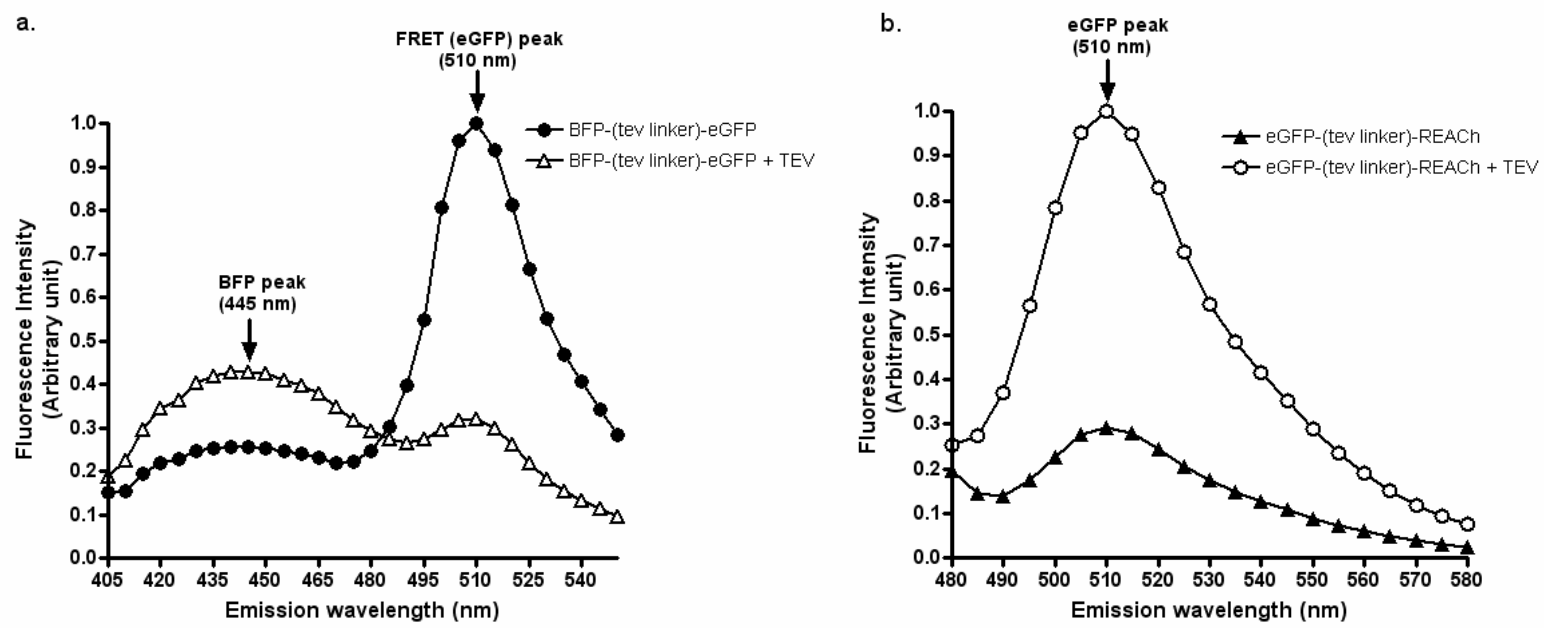

Fig. 2. Spectrofluorimetric analysis of recombinant FRET-based proteins. (a) BFP-(tev linker)-eGFP construct was incubated with 0.5 $\mu \mathrm{g}$ of recombinant TEV protease per $\mu \mathrm{g}$ of protein for 16 hours (open triangles) or without TEV protease (black circles). Emission spectra are shown for excitation at $365 \mathrm{~nm}$ that is optimal for BFP. The left arrow indicates the BFP emission peak; the right arrow indicates the eGFP emission peak that is the result of FRET from the BFP donor. (b) eGFP-(tev linker)-REACh construct was incubated with $0.5 \mu \mathrm{g}$ of recombinant TEV protease per $\mu \mathrm{g}$ of protein for 16 hours (open circles) or without TEV protease (black triangles). Emission spectra are shown for excitation at $457 \mathrm{~nm}$ that is optimal for eGFP. Arrow indicates the eGFP emission peak. All emission spectra are normalized to the maximum emission of the treated samples.

To apply our new optical substrate assay for the study of a theophylline-responsive riboswitch, we expressed TEV protease and FRET-based proteins in E. coli cells. We constructed two compatible plasmids for expression of these two proteins. The first plasmid contained the TEV protease gene that was cloned downstream of the synthetic riboswitch sequence based on published work by Lynch et al. ${ }^{5}$ The second plasmid was constructed by inserting of BFP-(tev linker)-eGFP or eGFP-(tev linker)-REACh coding sequences into the pHWG640 vector. Both plasmids were transformed into the same cells. Protein expression was performed by adding the corresponding inducers, and riboswitch activiation was achieved with $2.5 \mathrm{mM}$ theophylline. The theophylline-dependent increase in TEV protease activity was examined via the emission spectra of FRET-based proteins in cell cultures and lysates over a 6 hour time period after theophylline addition (Figure 3a, b c and d). The cells harboring riboswitch-TEV and BFP-(tev linker)-eGFP constructs did not show a significant difference in emission spectra of riboswitch "OFF" state (cells without theophylline) and riboswitch "ON" state (cells treated with theophylline) (Figure 3a). The eGFP emission at $510 \mathrm{~nm}$ was decreased in the riboswitch "ON" state as a result of FRET protein cleavage by produced TEV protease. However, changes in the emission signal at $445 \mathrm{~nm}$ were not observed due to the background fluorescence of cell culture components. To quantify the theophylline-dependent increase in TEV protease activity, we collected the emission spectra of cell lysates upon excitation at $365 \mathrm{~nm}$ (Figure 3b). Addition of theophylline resulted in a decrease in eGFP emission and a concurrent increase in BFP emission. This loss of FRET reflects the cleavage of BFP-(tev linker)-eGFP by TEV protease due to riboswitch activation in the presence of theophylline. Using BFP-(tev linker)-eGFP as a reporter, we determined a 1.5fold increase in TEV protease expression in the presence of theophylline.

When we used eGFP-(tev linker)-REACh as a reporter, the addition of theophylline led to a 6.7-fold increase in the eGFP emission in cell lysates as the result of the theophylline-responsive riboswitch activation (Figure $3 \mathrm{c}$ and d). The presence of significant background fluorescence in cell cultures reduces the difference between riboswitch "OFF" and 
riboswitch "ON" states. Thus, using the eGFP-REACh pair allowed us to examine and quantify the theophyllinedependent activation of the TEV protease with a higher dynamic rande and increased sensitivity, i.e., the elimination of acceptor fluorescence in eGFP-REACh pair led to a 4.5-fold improvement in signal-to-noise ratio compared to the BFPeGFP pair.

\section{BFP-eGFP}
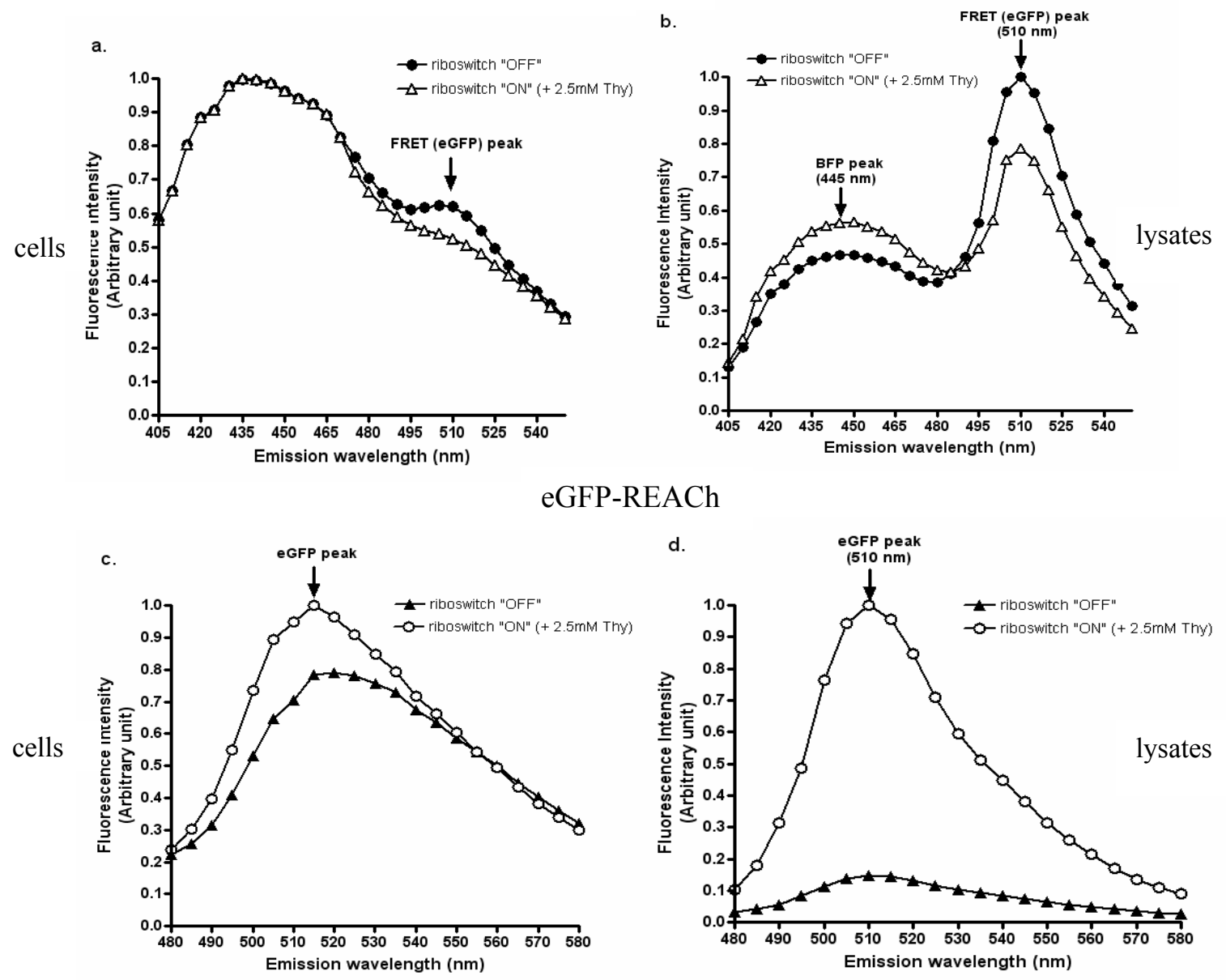

Fig. 3. Monitoring theophylline-responsive riboswitch activation in E. coli cells. (a, b) E. coli BL21 (DE3) cells expressing BFP-(tev linker)-eGFP and riboswitch-TEV protease were grown in LB media. $2.5 \mathrm{mM}$ theophylline was added (riboswitch "ON"), and cells were analyzed after 6 hours. Cells without theophylline treatment (riboswitch "OFF) were analyzed in parallel. The right arrow indicates the eGFP emission peak that is a result of FRET from the BFP donor. Riboswitch activation leads to production of TEV protease which cleaves FRET protein resulting in decreased eGFP emission. (c, d) E. coli TOP10 cells expressing eGFP-(tev linker)REACh and riboswitch-TEV protease were grown in LB media, treated and analyzed the same way as described above. Increased eGFP emission indicates cleavage of FRET protein by TEV protease as a result of riboswitch activation in response to theophylline. Emission spectra in panels a and b are excited at $365 \mathrm{~nm}$ and emission spectra in panels $\mathrm{c}$ and d are excited at $457 \mathrm{~nm}$. Panels a and c represent cell culture experiments; panels $b$ and $d$ represent cell lysate experiments.

Using our FRET-based reporter assays, we performed time course experiments to study riboswitch activation. The time course studies provided the best indication of switch dynamics and whether or not the switch was behaving as anticipated. As we expected, both optical assays showed similar riboswitch kinetic behavior (Figure 4a and c). The riboswitch "OFF" curves were very similar to the negative control data in which produced FRET-proteins were 
accumulated without cleavage by TEV protease. In contrast, the riboswitch "ON" curves were similar to positive control when TEV protease was expressed directly, i.e. riboswitch deleted from 5' untranslated region. The maximal difference between riboswitch "ON" and riboswitch "OFF" states for both FRET systems was observed 360 min after addition of theophylline (Figure $4 \mathrm{~b}$ and d). With the BFP-eGFP FRET pair, we were starting at a significant optical background of $\sim 0.35$ (Figure 4a). This was an unavoidable consequence of performing spectroscopic ratioing, common in FRET pairs with overlapping emission profiles. By using the eGFP-REACh FRET pair, we were able to start our kinetic measurement at a significantly lower optical background $(<0.01$; Figure $4 \mathrm{c})$. This allowed us to follow riboswitch activation kinetics in a more sensitive and accurate manner.

a.

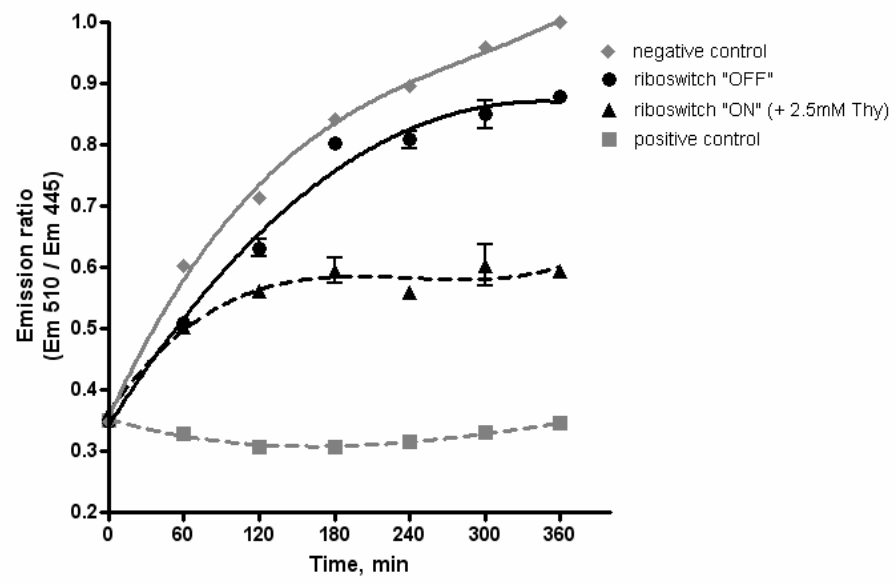

c.

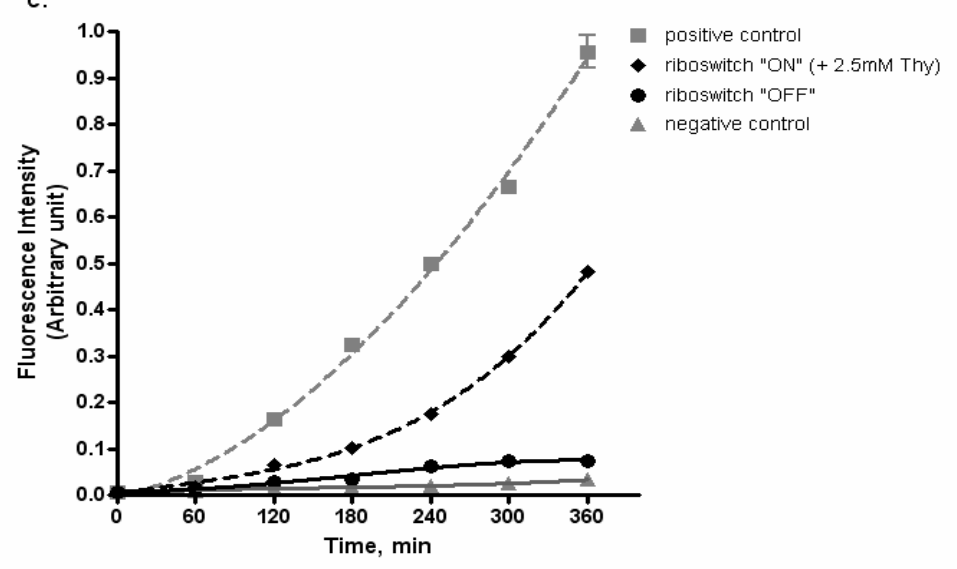

b.
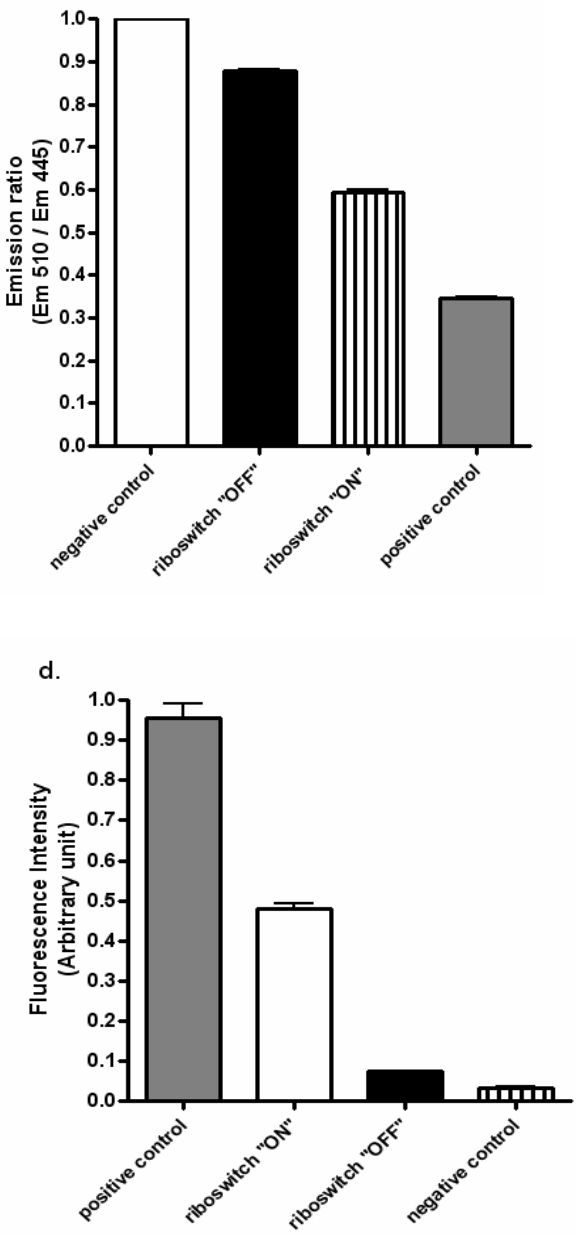

Fig. 4. Time course study of riboswitch activation in response to theophylline exposure. (a) BFP-(tev linker)-eGFP cleavage was monitored in cellular extracts of $E$. coli BL21 (DE3) cells harboring FRET-based protein (negative control) or FRET-based protein and one of the following constructs: TEV protease (positive control ); riboswitch-TEV protease (riboswitch "OFF"); riboswitch-TEV protease in the presence of theophylline (riboswitch "ON"). $2.5 \mathrm{mM}$ theophylline was added at time 0 and samples were collected at the indicated time points. (b) BFP-(tev linker)-eGFP cleavage as the result of riboswitch activation was monitored 360 min after theophylline addition. All data are normalized to the maximum emission ratio of the samples. (c) Emission at $510 \mathrm{~nm}$ was monitored in cellular extracts of E. coli TOP10 cells harboring eGFP-(tev linker)-REACh and one of the following constructs: TEV protease (positive control); riboswitch-TEV protease in the presence of theophylline (riboswitch "ON"); riboswitch-TEV protease (riboswitch "OFF"); riboswitch-TEVC151A inactive mutant in the presence of theophylline (negative control). 2.5 mM theophylline was added at time 0, and samples were collected at indicated time points. (d) Emission at $510 \mathrm{~nm}$ reflecting the cleavage of eGFP-(tev linker)REACh as the result of riboswitch activation was monitored $360 \mathrm{~min}$ after addition of theophylline. All data are normalized to the maximum emission of the samples. 


\section{CONCLUSIONS}

We have developed an optical reporter assay for monitoring of riboswitch activation which is based on the fluorescence resonance energy transfer between two fluorescent proteins. We have compaired two FRET-based reporting systems: one is based on the BFP-eGFP FRET pair and the second is composed of eGFP and REACh protein which is a nonfluorescent mutant of YFP. We have demonstrated the performance of an optical reporter assay by monitoring the activation of riboswitch-based sensor for detection of small molecule, theophylline.

We optimized the optical assay for this small molecule sensor using a low fluorescent background eGFP-REACh FRET protein pair, in order to achieve higher sensitivity to analyte exposure. The excitation wavelength needed for the BFPeGFP is unique allowing for reduced emission of eGFP signal due to loss of FRET; however the background fluorescence signal starts at a higher level, thus decreasing the signal-to-noise ratio. In contrast, the advantage of using an eGFP-REACh pair is the elimination of acceptor fluorescence which leads to a 4.5 -fold improvement in signal-tonoise ratio compared to the BFP-eGFP pair. By using the eGFP-REACh FRET construct, the degree of the enzymatic amplification was better demonstrated by quenching acceptor fluorescence background.

The amplification of signal via TEV protease cleavage is a benefit; however the burden of the cells to produce the protease substrate delays signal response to the analyte. In some settings, a time delay has beneficial applications. Reduction of this time delay may be achieved with direct expression of a fluorescent protein.

\section{ACKNOWLEDGMENTS}

This work was supported by funds provided by the Air Force Office of Scientific Research (AFOSR) and by the US Department of Defense High Performance Computing Modernization Program (HPCMP), under the High Performance Computing Software Applications Institutes (HSAI) initiative.

\section{DISCLAIMER}

The opinions or assertions contained herein are the private views of the authors and are not to be construed as official or as reflecting the views of the US Army, US Air Force or the US Department of Defense. This paper has been approved for public release with unlimited distribution.

\section{REFERENCES}

[1] Mandal, M., Breaker, R. R., Nature Rev. Mol. Cell. Biol. 5, 451-463 (2004).

[2] Tucker, B. J., Breaker, R. R., Curr. Opin. Struct. Biol. 15, 342-348 (2005).

[3] Nudler, E., Mironov, A. S., Trends Biochem. Sci. 29, 11-17 (2004).

[4] Winkler, W. C., Curr. Opin. Chem. Biol. 9, 594-602 (2005).

[5] Lynch, S. A., Desai, S. K., Sajja, H. K., Gallivan, J. P., Chem. Biol. 14, 173-184 (2007)

[6] Tyas, L., Brophy, V. A., Pope, A., Rivett, A. J., and Tavaré, J. M., EMBO Reports 1(3), $266-270$ (2000).

[7] Rehm, M., Düßmann, H., Jänicke, R. U., Tavaré, J. M., Kögel, D., and Prehn, J. H. M., J. Biol. Chem. 277(27), 24506-24514 (2002).

[8] Dong, M., Tepp, W. H., Johnson, E. A., and Chapman, E. R., PNAS 101(41), 14701-14706 (2004).

[9] Ganesan, S., Ameer-beg, S. M., Ng, T. T. C., Vojnovic, B., and Wouters, F. S., PNAS 103(11), 4089-4094 (2006).

[10] Marcello, A., Cinelli, R. A. G., Ferrari, A., Signorelli, A., Tyagi, M., Pellegrini, V., Beltram, F., and Giacca, M., J. Biol. Chem. 276(42), 39220-39225 (2001)

[11] Philipps, B., Hennecke, J., and Glockshuber, R., J. Mol. Biol. 327, 239-249 (2003) 
[12] Corradi, G. R. and Adamo, H. P., J. Biol. Chem. 282(49), 35440-35448 (2007)

[13] Kapust, R. B., Tozser, J., Fox, J. D., Anderson, D. E., Cherry, S., Copeland, T. D., Waugh, D. S., Protein Eng. 14, 993-1000 (2001).

[14] van den Berg, S., Löfdahl, P.-Å, Härd, T., Berglund, H., J. Biotechnol. 121, 291-298 (2006).

[15] Sambrook, J., Russell, D.W., Molecular Cloning : Laboratory Manual. $3^{\text {rd }}$ ed.; Cold Spring Harbor Laboratory Press: Cold Spring Harbor, N.Y. (2001).

[16] QIAexpress ${ }^{\circledR}$ Ni-NTA Fast Start Handbook, QIAGEN (2006)

[17] Heim, R. and Tsien, R., Curr. Biol. 6, 1178-1182 (1996)

[18] Mitra, R., Silva, C., and Youvan, D., Gene 173, 13-17 (1996) 